\title{
Comparison of Adherence and CD4 counts of HIV patients on various ART Regimens
}

\author{
Adiga $\mathbf{S}^{1}$, Adiga $\mathbf{U}^{2}$, Malawadi $\mathrm{BN}^{3}$ \\ ${ }^{1}$ Dr Sachidananda Adiga, Associate Professor, Department of Pharmacology, ${ }^{2}$ Dr Usha Adiga, Associate Professor, \\ Department of Biochemistry, ${ }^{3}$ Dr Malawadi BN, Assistant Professor, Department of Biochemistry, all are affiliated with \\ Karwar Institute of Medical Sciences, Karwar. Karnataka, India.
}

Address for Correspondence: Dr. Sachidananda Adiga, Associate Professor, Department of Pharmacology, Karwar Institute of Medical Sciences, Karwar. Karnataka, India. E-mail : adigaiscool@yahoo.com

\begin{abstract}
Introduction: Inspite of HAART, it has been found that viral replication persists even when viral load is undetectable. Adherence has been reported to be positively associated with CD4 count in various settings. Objectives of our study were comparison of basal CD4 count of HIV patients on various ART regimens with that at six months and one year as well as finding correlation between percentage adherence to ART and CD4 counts at six months and one year. We also aimed to compare percentage adherence and CD4 counts of patients on various ART regimens. Methodology: A retrospective observational study was conducted on $63 \mathrm{HIV}$ patients, receiving four antiretroviral regimens (AZT/3TC/NVP, AZT/3TC/EFV, d4T/3TC/NVP and d4T / 3TC / EFV). Patient demography, drug regimen, baseline CD4 counts, serial CD4 counts (every six months), and adherence to ART (monthly) were recorded. Comparison of basal CD4 counts with that at six months and one year was done. Comparison of CD4 count and adherence were done between the groups. Results: An extremely significant elevation was seen in CD4 counts at six months and at one year $(p<0.0001)$. Linear regression showed a significant positive correlation $(p=0.037)$ between adherence and CD4 counts at one year. There was no significant difference in percentage adherence and CD4 counts between four groups. Conclusion: We conclude that optimal CD4 levels were obtained in patients on all four different regimens. However percentage adherence was suboptimal in all groups which caution to take appropriate measures to achieve optimal drug adherence.
\end{abstract}

Key words: Adherence, Antiretroviral therapy, CD4 Count, HIV Infection

\section{Introduction}

The treatment of HIV infection and AIDS is complex because of many reasons. The complexity of ART regimens used, duration of treatment, adherence to treatment, drug toxicities and opportunistic infections associated are the main contributing factors. Several studies have shown that nearly $25 \%$ of all patients discontinue their initial HAART regimen because of treatment failure, toxic effects or noncompliance within the first eight months of therapy [1, 2]. Many studies have shown that adherence to treatment is most important factor in deciding the progression of the disease and there are many factors associated with this [3-7]. The Indian national AIDS control organization guidelines recommended the use of two nucleoside

Manuscript received $14^{\text {th }}$ June 2016

Reviewed: $25^{\text {th }}$ June 2016

Author Corrected: $4^{\text {th }}$ July 2016

Accepted for Publication $15^{\text {th }}$ July 2016 reverse transcriptase inhibitors (NRTI) in combination with a non-nucleoside reverse transcriptase (NNRTI) in India. Zidovudine and lamivudine in combination with either nevirapine or efavirenz and stavudine, lamivudine in combination with nevirapine or efavirenz are commonly used regimens.

\section{Primary objectives}

1. Comparison of basal CD4 count of HIV patients on various ART regimens with that at six months and one year.

2. Correlate percentage adherence to ART and CD4 counts at six months and one year.

Secondary objective- Comparison of CD4 counts and percentage adherence to ART, among patients receiving different ART regimens. 


\section{Patients and Methods}

A retrospective observational study on 63 adult HIV patients, receiving various antiretroviral regimens was conducted in a teaching hospital attached to Karwar Institute of Medical Sciences, Karwar Karnataka, India. Institutional ethics committee approval was sought. Data of patients who were diagnosed to be HIV positive, receiving HAART and were attending the hospital for regular follow up monthly was collected. Patients were evaluated during their scheduled follow up visits in detail, by measuring CD4 counts, hemogram and other laboratory parameters serially once in six months. Adherence to the regimen was assessed monthly.

Inclusion Criteria: Patients receiving one of the above mentioned regimens at least for one year.

Exclusion: Data of patients with less than one year of treatment, deaths within 1 year were excluded.

Data Collection- Data was extracted from Patient's medical records using data collection form. Patient demography such as age, gender, medication prescribed (drug regimen), baseline CD4 cell counts, serially monitored CD4 count values (once in six months), adherence to ART by pill count method (monthly) were recorded.

Patients' data was categorized into four groups based on the type of regimen.

Group 1: zidovudine, lamivudine, nevirapine (ZLN) AZT/3TC/NVP

Group 2: zidovudine, lamivudine, efavirenz (ZLE) AZT/3TC/EFV

Group 3: stavudine, lamivudine, nevirapine (SLN) d4T/3TC/NVP

Group 4: stavudine, lamivudine, efavirenz (SLE) d4T / 3TC / EFV

Standard drug dosages of the nucleoside reverse transcriptase inhibitors (NRTIs) were, zidovudine (AZT) $300 \mathrm{mg}$ twice daily, lamivudine (3TC) $150 \mathrm{mg}$ twice daily or $300 \mathrm{mg}$ once daily, stavudine (d4T) $60 \mathrm{mg}$ daily. Dosages of non nucleoside reverse transcriptase inhibitors (NNRTIs) were nevirapine (NVP) $200 \mathrm{mg}$ once daily for a 2-week lead-in period and then as $200 \mathrm{mg}$ twice daily and efavirenz (EFV) $600 \mathrm{mg}$ once daily .Demographic profile of patients are given in Table 1.

Table-1: Demographic profile of patients on different regimens.

\begin{tabular}{|c|c|c|c|c|c|}
\hline & Group 1(ZLN) & Group 2(ZLE) & Group 3(SLN) & Group 4(SLE) \\
\hline \multicolumn{2}{|c|}{ Number of patients } & 15 & 18 & 14 & 16 \\
\hline \multirow{2}{*}{ Gender: } & Males & $53.33 \%$ & $50 \%$ & $35.71 \%$ & $68.75 \%$ \\
\cline { 2 - 6 } & Females & $46.67 \%$ & $50 \%$ & $64.29 \%$ & $31.25 \%$ \\
\hline \multicolumn{2}{|c|}{ Age (years) } & $35.67 \pm 2.38$ & $39.39 \pm 1.89$ & $31.92 \pm 4.2$ & $39.38 \pm 2.54$ \\
\hline \multicolumn{2}{|c|}{ Body weight (kg) } & $51.13 \pm 3.06$ & $46.4 \pm 1.95$ & $35.29 \pm 3.65$ & $46.69 \pm 3.09$ \\
\hline
\end{tabular}

Basal CD4 count and improvement in CD4 counts in subsequent follow up visits and adherence to ART are the tool to compare and measure the effectiveness of the regimens. CD4 count was measured by flow cytometric method. Percentage drug adherence was calculated by pill count method, which is a process oriented indicator. Adherence assessment was done monthly and mean was taken at six months and one year.

Statistical analysis was done by Graphpad Prism-7 statistical software. Student's paired ' $t$ ' test was done to compare basal CD4 counts with that at six months and one year separately. Linear regression analysis was carried out to find the correlation between adherences and follow up CD4 counts, taking CD4 count as dependent variable. One way ANOVA test was used for the comparison of basal as well as CD4 counts at six months and one year, between as well as within the groups. Statistical significance was fixed at level 0.05 .

\section{Results}

An extremely significant $(\mathrm{p}=0.0000)$ elevation was seen in CD4 counts at 6 months as well as at 1 year $(\mathrm{p}<0.0001)$ as shown in Table 2. When CD4 counts of 6 months were compared with that at 1 year, a less significant elevation (0.0273) was obtained. Linear regression showed a significant positive correlation $(p=0.037)$ between adherence and CD4 counts at 1 year $(\mathrm{R}=0.13, \mathrm{R} 2=0.02)$. 
Table-2: Comparison of CD4 counts and percentage adherence in HIV patients.

\begin{tabular}{|c|c|c|c|}
\hline & $\begin{array}{c}\text { Before starting (A) ART(N=63) } \\
\text { Mean } \pm \text { SEM }\end{array}$ & $\begin{array}{c}\text { At 6 months (B) } \\
(\mathrm{N}=63) \\
\text { Mean } \pm \text { SEM }\end{array}$ & $\begin{array}{c}\text { At 1 year }(\mathrm{C}) \\
(\mathrm{N}=63) \\
\text { Mean } \pm \text { SEM }\end{array}$ \\
\hline CD4 count & $230.91 \pm 26.79(\mathrm{~A})$ & $391.65 \pm 34.03(\mathrm{~B})$ & $458.86 \pm 42.38(\mathrm{C})$ \\
\hline Median & 164 & 360 & 373 \\
\hline \% of Adherence & ------- & $93.28 \pm 1.36$ & $92.91 \pm 3.5$ \\
\hline
\end{tabular}

A Vs B : $p=0.0000$, extremely significant

A Vs C : p $<0.0001$, highly significant

B Vs C : $\mathrm{p}=0.0273$, significant

We did not find any significant difference in the CD4 counts (basal, six months and one year) between the four treatment regimens. However a highly significant $(\mathrm{p}=0.0021)$ elevation was seen in CD4 counts in group 1 . A comparatively less significant elevation was seen in group $2(\mathrm{p}=0.0461)$. No significant improvement was seen in group 3 . A highly significant elevation was also seen in group $4(\mathrm{p}=0.006)$. Comparison of CD4 counts between the groups is given in Table 3.

Table-3: Comparison of percentage adherence and CD4 counts between different groups.

\begin{tabular}{|c|c|c|c|c|}
\hline & $\begin{array}{c}\text { Group 1 (ZLN) } \\
(\mathbf{N = 1 5})\end{array}$ & $\begin{array}{c}\text { Group 2 (ZLE) } \\
(\mathbf{N = 1 8})\end{array}$ & $\begin{array}{c}\text { Group 3 (SLN) } \\
(\mathbf{N = 1 4 )}\end{array}$ & $\begin{array}{c}\text { Group 4 (SLE) } \\
(\mathbf{N = 1 1 6})\end{array}$ \\
\hline Basal CD4 count & $225.8 \pm 28.9$ & $196.22 \pm 41.53$ & $363.21 \pm 89.89$ & $158.94 \pm 34.17$ \\
\hline CD4 count at 6 months & $406.53 \pm 62.11$ & $357 \pm 73.43$ & $482.57 \pm 86.22$ & $337.13 \pm 46.72$ \\
\hline CD4 count at 1 year & $568.66 \pm 86.98$ & $397.51 \pm 70.49$ & $517.2 \pm 122.26$ & $373.75 \pm 57.3$ \\
\hline P value comparing all 3 CD4 counts & $0.0021^{* *}$ & $0.0461^{*}$ & 0.54 & $0.006^{* *}$ \\
\hline $\begin{array}{c}\text { Percentage of adherence at 6 } \\
\text { months }\end{array}$ & $93.71 \pm 1.8$ & $95.52 \pm 0.32$ & $94.52 \pm 0.8$ & $94.25 \pm 1.06$ \\
\hline Percentage adherence at 1 year & $95.21 \pm 1.35$ & $95.86 \pm 0.5$ & $95.19 \pm 0.74$ & $94.38 \pm 0.6$ \\
\hline
\end{tabular}

*significant

**highly significant

There was no significant difference in percentage of adherence between the groups as well as within individual groups at six months and one year. No significant gender difference was observed in percentage adherence in our study.

\section{Discussion}

It is obvious from our findings that, CD4 counts were elevated during their initial one year treatment period. Extent of elevation was higher in the first six months as compared to the next six months. Positive correlation between percentage adherence and CD4 counts at one year suggests that, better the drug adherence, higher is the CD4 count. Our study is supported by a study by Ghate et al which suggests a significant elevation in CD4 counts if adherence is high [8]. Similar results were expressed in a South African study [9]. A study by Paterson and collegues suggest that below 95\%, adherence rate independently determines viral load [10].
WHO bulletin suggests that, response to ART therapy is said to be optimal if rise in median CD4 count is more than 50 cells $/ \mu \mathrm{l}$ at six months and more than 100 cells/ $\mu 1$ at one year [11]. As per these guidelines, our patients have shown optimal response to ART therapy. A few studies agree with our report in this regard $[12,13]$.

Various Indian studies have reported that adherence rate ranges from 41\%-97.3\% [14-19] Compared to majority of these studies, adherence rate in our study is better. Even though we could achieve optimal CD4 counts, 
percentage adherence is not more than $95 \%$ in all the groups. The national ART program in India considers adherence to be optimal if percentage adherence is more than or equal to $95 \%$. This calls for a need to improve drug adherence in our patients. This not only improves immunological response and viral suppression, but also reduces drug resistant pathogens [20].

We did not find any significant difference in CD4 counts at six months and one year, among the four groups. A report by Joly et al supports our result [21]. This study suggests that regimens containing zidovudine exhibited same levels of antiviral activities as that containing stavudine. Based on this particular point of our result, we cannot decide which regimen is better. Values of percentage adherence to the regimens also are not helpful as they did not differ significantly between the groups.

However $\mathrm{P}$ values obtained by comparing basal, six months and one year CD4 counts within individual groups (Table 3) supports the extent of elevation in CD4 count. 2.5 times elevation of CD4 count was observed in patients receiving ZLN regimen (group 1) at the end of one year. Extent of CD4 count elevation was 2.35 times in patients on SLE (group 4).

Minimum elevation in CD4 count (1.4 times) was in patients receiving ZLE (group 2) whereas those on SLN (group 3) had least extent of elevation. Based on these findings, we can suggest that ZLN is the preferred drug in resource limited settings.

Missing scheduled follow up appointments and drug toxicities are responsible for suboptimal drug adherence $[15,22]$. It is not just national ART program, but also patients and their family, health care providers and community have an important role in achieving optimal adherence rate.

Limitations of our study-Small sample size and method used for assessment of adherence are the limitations of our study. It has been suggested that pill count method is not the best standard for assessment of adherence as it does not match with self-reporting by patients.

More than one method has been recommended to achieve more accurate assessment of adherence [23]. However adherence assessment in our study was better than several studies which have obtained one time adherence $[14,15,16,17]$.

\section{Conclusion}

We conclude that optimal CD4 levels were obtained in patients on all four different regimens. However percentage adherence was suboptimal in all groups which cautions measures to be taken to achieve optimal drug adherence.

Acknowledgements- We thank Dr Anand Pawaskar, Medical Officer, ART center, Karwar for the support.

Funding: Nil, Conflict of interest: None initiated. Permission from IRB: Yes

\section{References}

1. d'Arminio Monforte A, Lepri AC, Rezza G, Pezzoti $\mathrm{P}$, Antinori A, Phillips AN, et al. Insights into the reasons for discontinuation of the first highly active antretroviral therapy (HAART) regimen in a cohort of antiretroviral naove patients: Italian cohort of antiretroviral Naove patients. AIDS 2000; 14(5):499507.

2. Lucas GM, Chaisson RE, Moore RD. Highly active antiretroviral therapy in a large urban clinic: Risk factors for virologic failure and adverse drug reactions. Ann Intern Med 1999; 131(2):81-7.

3. Wood E, Hogg RS, Yip B, Harrigan PR, O'Shaughnessy MV, Montaner JS. Effect of Medication Adherence on Survival of HIV-Infected Adults Who Start Highly Active Antiretroviral Therapy When the CD4+ Cell Count Is 0.200 to $0.350 \times 109$ cells/L. Annals of Internal Medicine 2003; 139 (10): 810-6.

4. Abaasa AM, Todd J, Ekoru K, Kalyango JN, Levin J, Odeke E et al. Good adherence to HAART and improved survival in a community HIV/AIDS treatment and care programme: the experience of The AIDS Support Organization (TASO), Kampala, Uganda. BMC Health Serv Res 2008;8: 241 .doi: 10.1186/14726963-8-241.

5. Mills EJ, Bakanda C, Birungi J, Chan K, Ford N, Cooper CL et al. Life expectancy of persons receiving combination antiretroviral therapy in low-income countries: a cohort analysis from Uganda. Ann Intern Med 2011; 155(4): 209-216 .doi: 10.7326/0003-4819155-4-201108160-00358.

6. Chi BH, Cantrell RA, Zulu I, Mulenga LB, Levy JW, Tamba Tamba BC et al. Adherence to first-line 
antiretroviral therapy affects non-virologic outcomes among patients on treatment for more than 12 months in Lusaka, Zambia. Int J Epidemiol2009;38 (3): 746-756 .doi: 10.1093/ije/dyp004.

7. Reda AA, Biadgilign S. Determinants of Adherence to Antiretroviral Therapy among HIV-Infected Patients in Africa. AIDS Research and Treatment 2012;2012: 8.doi: 10.1155/2012/574656.

8.Manisha Ghate, Srikanth Tripathy, Raman Gangakhedkar, Madhuri Thakar, Jayanta Bhattacharya, Ipsita Choudhury et al. Use of first line antiretroviral therapy from a free ART programme clinic in Pune, India- A preliminary report. Indian J Med Res 2013; 137: 942-949.

9. Coetzee D, Hildebrand K, Boulle A, Maartens G, Louis F, Labatala V, et al. Outcomes after two years of providing antiretroviral treatment in Khayelitsha, South Africa. AIDS 2004; 18(6): 887-95.

10. Paterson DL, Potoski B, Capitano B. Measurement of adherence to antiretro viral medications. J of Acq immun def synd 2002;31(3):S103-s106.

11. WHO/UNAIDS/IAS. Safe and Effective Use of Antiretroviral Treatment in Adults With Particular Reference to Resource Limited Settings. Munderi P, Prag E, Vella S, editors. Geneva, Switzerland: WHO/ AIDS 2000: 1-31.

12. Sharma SK, Dhooria S, Prasad KT, George N, Ranjan S, Gupta D, et al. Outcomes of antiretroviral therapy in a northern Indian urban clinic. Bull World Health Organ2010; 88: 222-6.

13. Bachani D, Garg R, Rewari BB, Hegg L, Rajasekaran S, Deshpande A, et al. Two-year treatment outcomes of patients enrolled in India's national firstline antiretroviral therapy programme. Natl Med J India 2010; 23(1) : 7-12.

14. Shet A, DeCosta A, Heylen E, Shastri S, Chandy S, EkstrandM. High rates of adherence and treatment success in a public and public-private HIV clinic in India: potential benefits of standardized national care delivery systems. BMC Health Ser Res2011; 11: 277. doi: 10.1186/1472-6963-11-277

15. Venkatesh KK, Srikrishnan AK, Mayer KH. Kumarasamy N, Raminani S, Thamburaj E. et al.
Predictors of non adherence to highly active antiretroviral therapy among HIV-infected South Indians in clinical care: Implications for developing adherence interventions in resource-limited settings. AIDS Patient Care STDs 2010; 24(12) : 795-803.doi: 10.1089/apc.2010.0153.

16. Cauldbeck MB, O' Connor C, O'Connor MB, Saunders JA, Rao B, Mallesh VG, et al. Adherence to anti-retroviral therapy among HIV patients in Bangalore, India. AIDS Res Ther 2009; 6 : 7.doi: 10. 1186/1742-6405-6-7

17. Shah B, Walshe L, Saple DG,Mehta SH, Ramnani JP, Kharkar RD, et al. Adherence to antiretroviral therapy and virologic suppression among HIV-infected persons receiving care in private clinics in Mumbai, India. Clin Infect Dis2007; 44(9): 1235-44.DOI:10. 1086/513429

18. Sharma M, Singh RR, Laishram P,Kumar B, Nanao $\mathrm{H}$, Sharma $\mathrm{C}$, et al. Access, adherence, quality and impact of ARV provision to current and ex-injecting drug users in Manipur (India): an initial assessment. Int J Drug Policy2007; 18 (4): 319-25.DOI:10. 1016/j. drugpo.2007.04.001

19. Safren S, Kumarasamy N, James R, Raminani S, Solomon S Mayer KH. ART adherence, demographic variables and CD4 outcome among HIV-positive patients on antiretroviral therapy in Chennai, India. AIDS Care 2005; 17 (7): 853-62. DOI:10.1080/ 09540120500038439

20. Steel G, Nwokike J, Joshi M. Development of a multi-method tool to measure ART adherence in resource-constrained settings: the South Africa experience. Submitted to the U.S. Agency for International Development by the Rational Pharmaceutical Management Plus Program. Arlington, VA: Management Sciences for Health; 2007.

21. Joly V, Flandre P, Meiffredy V, Brun-Vezinet F, Gastaut JA, Goujard C et al. Efficacy of Zidovudine Compared to Stavudine, Both in Combination with Lamivudine and Indinavir, in Human Immunodeficiency Virus-Infected NucleosideExperienced Patients with No Prior Exposure to Lamivudine, Stavudine, or Protease Inhibitors (Novavir Trial). Antimicrob agents chemother. 2002; 46(6): 1906-1913. 
22. Bachani D, Garg R, Rewari BB, .Hegg L, Rajasekaran S, Deshpande A, et al. Two-year treatment outcomes of patients enrolled in India's national first- line antiretroviral therapy programme. Natl Med J India 2010; 23 (1): 7-12.

\section{How to cite this article?}

Adiga S, Adiga U, Malawadi BN. Comparison of Adherence and CD4 counts of HIV patients on various ART Regimens. Int J Med Res Rev 2016;4 (7):1138-1143.doi: 10.17511/ijmrr.2016.i07.12. 